\title{
Emprendimiento digital femenino para el desarrollo social y económico: características y barreras en España
}

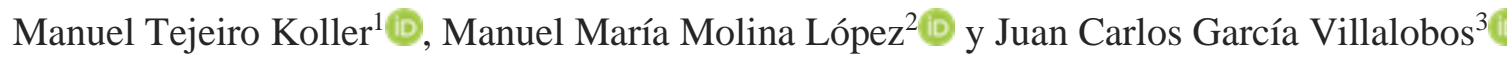

Recibido: 20 de enero de 2021 / Aceptado: 2 de febrero de 2021 / Publicado: 27 de abril de 2021

Resumen. El presente estudio centra su atención en un tipo particular de emprendimiento: el emprendimiento digital iniciado por mujeres. Este tipo de creación de empresas tiene un interés particular por su alto impacto en el desarrollo económico y la dinamización de las economías desarrolladas; y en muchos casos además por su intensa orientación social. Aquí resulta especialmente relevante encontrar una paridad entre hombres y mujeres a la hora de emprender, ya que, de lo contrario, no se obtendría un desarrollo inclusivo, ni se explotaría todo el potencial emprendedor de una sociedad. Tras una descripción de las características particulares tanto del emprendimiento digital, como del femenino, se revisan las barreras a las que comúnmente se enfrenta cada uno de ellos. Mediante un estudio empírico, que logró recopilar la respuesta de 161 emprendedoras digitales en España, se procede a contrastar las barreras identificadas en la literatura con la realidad empresarial. Se pueden confirmar las dificultades que comúnmente enfrentan emprendimientos digitales, al igual que femeninos, en lo referente a las dificultades para encontrar financiación. Sin embargo, otras barreras, tales como el rol de la mujer en la familia, parecen tener menor relevancia en este tipo de emprendimientos. Finalmente, se ofrecen conclusiones generales, en las que se destacan los puntos de actuación para posibles políticas de fomento del emprendimiento digital femenino.

Palabras clave: Emprendimiento; Digital; Social; Mujer; Barreras.

Claves Econlit: L26; M13.

\section{[en] Digital female entrepreneurship for social and economic development: characteristics} and barriers in Spain

\begin{abstract}
This study focuses on a particular type of entrepreneurship. Digital entrepreneurship initiated by women. This type of business creation is of particular interest because of its high impact on economic development and the dynamization of developed economies, in many cases with relevant social orientation. Here it is especially relevant to find parity between men and women when it comes to entrepreneurship, since otherwise, inclusive development would not be achieved, nor would all the entrepreneurial potential of a society be exploited. After a description of the particular characteristics of both, digital and female entrepreneurship, we review the barriers that commonly face each of them. Through an empirical study, which compiled the responses of 161 digital entrepreneurs in Spain, we proceed to contrast the identified barriers in the literature with the business reality. The difficulties commonly faced by digital entrepreneurs can be confirmed, as well as those faced by women, in terms of difficulties in finding financing. However, other barriers, such as the role of women in the family, seem to have less relevance in this type of enterprises. Finally, general conclusions are offered, in which the points of action for possible policies to promote female digital entrepreneurship are highlighted.
\end{abstract}

Keywords: Entrepreneurship; Digital; Social; Women; Barriers.

Sumario. 1. Introducción. 2. Definición de empresas digitales, 3. Principales barreras al emprendimiento digital. 4. Principales barreras de las emprendedoras. 5. Objetivos y metodologías. 6. Resultados. 7. Conclusiones. 8. Limitaciones y estudios futuros. 9. Referencias bibliográficas.

Cómo citar. Tejeiro Koller, M.; Molina López, M.M.; García Villalobos, J.C. (2021) Emprendimiento digital femenino para el desarrollo social y económico: características y barreras en España. REVESCO. Revista de Estudios Cooperativos, vol. 138 , e75561. https://dx.doi.org/10.5209/reve.75561.

1 Universidad San Pablo CEU, España.

Dirección de correo electrónico: manuel.tejeirokoller@ceu.es.

2 Universidad San Pablo CEU, España.

Dirección de correo electrónico: molinalopez@ceu.es.

3 Universidad San Pablo CEU, España.

Dirección de correo electrónico: garvil@ceu.es. 


\section{Introducción}

El emprendimiento es uno de los motores de cualquier economía (Minniti, 2012; Meyer \& de-Jongh, 2018; Acs, Audretsch, Braunerhjelm, \& Carlsson, 2012). Contribuye a la difusión de los avances científicos, a la renovación del tejido empresarial y la creación de nuevos puestos de trabajo (Galindo \& Méndez-Picazo, 2013; Jain \& Saiyed-Wajid, 2013). De entre todos los tipos de emprendimiento, el basado en las tecnologías avanzadas es considerado de mayor impacto para economías desarrolladas (Wong et al., 2005). El estudio global sobre el emprendimiento, Global Entrepreneurship Monitor (GEM), designa aquel tipo de emprendimiento con mayor impacto como el innovador y de alto impacto (High-growth entrepreneurship), entendiéndose éste como dinamizador de la economía, difusor de avances científicos y creadores de empleo (Bosma \& Kelly, 2019). Se puede afirmar, por tanto, que el desarrollo inclusivo de las economías desarrolladas será impulsado en gran medida por el aprovechamiento de empresas innovadoras que además pueden revestir diferentes formas jurídicas como las sociedades cooperativas ${ }^{4}$. Asimismo, si se quiere explotar al máximo su potencial y realmente igualar el campo de juego para todos los participantes, es esencial alcanzar una participación igualitaria entre hombres y mujeres emprendedoras (Minniti, 2010). Para ello es importante diagnosticar en qué medida participan las mujeres en el emprendimiento digital y cuáles son sus barreas para acceder a este tipo de emprendimientos de alto impacto. Esta es la contribución que quiere hacer el presente estudio 5 .

La brecha de género en materia de emprendimiento parece ir reduciéndose en España de forma más notable que en otros países ${ }^{6}$. Así pues, según el Informe Mundial GEM de 2018/19, por cada 10 hombres emprendedores en nuestro país hay 9 mujeres emprendedoras, siendo la relación en el total de la Unión Europea de 6 mujeres por cada 10 hombres. De acuerdo con el Informe, España se encuentra en el puesto 16 mundial para emprender un negocio, por encima de países como Alemania $\left(19^{\circ}\right)$, Japón $\left(20^{\circ}\right)$ o Reino Unido $\left(30^{\circ}\right)$. Dicho ranking es encabezado por Qatar, ocupando EE. UU. el sexto puesto (GEM, 2019).

La diferencia de género en materia de emprendimiento se podría explicar por, entre otros, los inferiores capitales de partida con los que cuentan las mujeres y al menor acceso al crédito al que suelen enfrentarse (Brooks et al., 2014; García Villalobos et al. 2019; GEM, 2019). A estas barreras sistémicas a las que se enfrentan las mujeres habría que añadir las barreras sociales que han podido promover, como resultado de la Teoría de los Roles de Género, que las mujeres sean menos arriesgadas o ambiciosas que los hombres (González-Pernía et al., 2012).

A su vez, otros factores como la conciliación laboral y familiar tienden a potenciar esta brecha, ya que la mujer sigue siendo, en muchas ocasiones, la principal encargada de las labores del hogar y del cuidado de los hijos o padres. Así, las mujeres tienden a dedicar más horas que los hombres a las actividades familiares, lo que da lugar a una desigualdad de oportunidades profesionales y de ingresos (Straub, 2007).

En este contexto de eventual desigualdad es donde el emprendimiento femenino, digital en nuestro caso, participa en la creación de valor social marcadamente sostenible que como dicen Sánchez, Martín, Bel y Lejarriaga, (2018) puede manifestarse de diferentes formas: actuando respetuosamente con el medio ambiente, favoreciendo la inclusión social, por ejemplo, atendiendo a colectivos que requieren de una atención social no cubierta suficientemente por el Estado o a través fórmulas que contribuyen a la creación de empleo estable, al desarrollo territorial y a la cohesión social. En este sentido, hay autores que reconocen diferencias de género en tanto que las mujeres se muestran más propensas a la búsqueda del valor social, con mayor inquietud por iniciativas de componente medioambiental, que los hombres más enfocados en la parte exclusivamente económica (Justo, 2017).

En materia de emprendimiento femenino en el ámbito tecnológico, la brecha anteriormente comentada se acentúa. En una muestra de 141 emprendedores tecnológicos en la Comunidad de Madrid, se ha podido constatar que el $84 \%$ eran hombres, frente a tan solo el 16\% de mujeres (Morales-Alonso et al., 2016). Si bien es cierto que no se dispone en la actualidad de amplia información acerca de la distribución de géneros en el ámbito del emprendimiento digital, este dato sobre la baja presencia de mujeres aporta una aproximación. Además, las mujeres muestran una participación relativamente menor en la actividad innovadora, siendo en otros países europeos tan solo entre el $15 \%$ y el $29 \%$ mujeres de las autoras de patentes (Naldi et al. 2005). Países del sur como España, Italia y Francia tienen una puntuación más alta que los países del norte, como Gran Bretaña o Alemania, pudiendo deberse este hecho a la mayor presencia de investigadoras en el ámbito privado en estos últimos países (Naldi et al. 2005).

\footnotetext{
Un estudio sobre el impacto del emprendimiento cooperativo en la economía sostenible puede verse en: Fernandez-Guadaño, J. Lopez-Millan, M., Sarria-Pedroza, J. (2020) Cooperative Entrepreneurship Model for Sustainable Development. Sustainability, $12,5462$.

Este trabajo forma parte del proyecto competitivo I+D+i FEM2017-83006-R, financiado por AEI/FEDER UE, adscrito a la Cátedra CEU-Mutua Madrileña: 060516-USPMM-02/17. Los resultados obtenidos han sido posibles gracias al convenio de colaboración entre la Universidad CEU San Pablo y Women Startup Community.

6 Para un análisis del emprendimiento femenino en España también puede consultarse: Montero González, B. y Camacho Ballesta, J.A. (2018). Caracterización del emprendimiento femenino en España: Una visión de conjunto. REVESCO. Revista de Estudios Cooperativos, Tercer Cuatrimestre, $N^{o} 129$, pp. 39-65. DOI: 10.5209/REVE.61936.
} 
Asimismo, de acuerdo con el Informe 'Mapa del emprendimiento 2019' elaborado por Spain StartupSouth Summit, en España el 39\% de las startups tienen, al menos, una mujer emprendedora dentro de su equipo fundador, siendo la media en Europa del $36 \%$ y del $50 \%$ en Latinoamérica. A su vez, y en lo que respecta al total de startups, España mantiene ese liderazgo con un porcentaje del $16 \%$ de startups creadas por mujeres, siendo la media de Europa del $13 \%$ según el portal EU-Startups.

Un ámbito que no ha sido estudiado es el emprendimiento digital, principalmente por la dificultad de identificar este tipo de empresas, que se caracterizan principalmente por apoyarse en tecnología digital, como la cloud computing, big data o la inteligencia artificial para desarrollar su modelo de negocio (definidas en mayor detalle en la siguiente sección). Así pues, se hace interesante estudiar el papel que tiene la mujer en este tipo de emprendimientos. El presente estudio caracteriza, a través de un cuestionario, el emprendimiento digital en España, analizando las principales barreras y retos a los que se enfrentan las mujeres emprendedoras, aportando recomendaciones para distintas políticas de emprendimiento y de desarrollo económico y territorial, en este último caso con gran potencial del emprendimiento como es reconocido de forma expresa por las propias instituciones europeas (Enciso, M.; Gómez, L. y Mugarra, A., 2012).

\section{Definición de empresas digitales}

Las empresas digitales se incluyen dentro de las llamadas "empresas de base tecnológica" o nuevas empresas de base tecnológica (NEBTs), debido a que basan su actividad en una tecnología. Empresas de base tecnológica son "aquellas de pequeño tamaño y propiedad independiente que operan en sectores relacionados con las nuevas tecnologías" (Storey y Tether ,1998). A su vez, los autores indican que estas empresas tienen una baja antigüedad al ser, en su mayoría, empresas de reciente creación, que aprovechan tecnologías novedosas. Estas NEBTs suelen caracterizarse por un alto potencial de crecimiento (Almus y Nerlinger, 1999) y una alta progresión de su valor añadido, especialmente las más jóvenes (Motohashi, 2005), de lo que se deriva el alto impacto identificado en el GEM.

Más específicamente, podemos diferenciar entre emprendimiento tecnológico, emprendimiento tecnológico digital y emprendimiento digital (Giones y Brem, 2017). En primer lugar, se identifica el emprendimiento tecnológico con la creación de nuevos productos basados en el avance en la investigación, avances con base científica a través de conocimiento específico en un campo académico y activando un nicho de mercado para ello. La financiación se consigue generalmente a través de fondos públicos o venture capital atraído por la propiedad intelectual (ejemplo, Grafeno) (Giones y Brem, 2017).

En segundo lugar, y con relación al emprendimiento tecnológico digital, se identifica con nuevos productos basados solamente en las tecnologías de la comunicación (TIC), creando para ello dispositivos inteligentes y utilizando las posibilidades del Internet de las Cosas y de otras tecnologías ya existentes. La financiación la encuentran habitualmente por medio de Business Angels, mercados financieros, capital semilla y capital riesgo o crowdfunding (ejemplo, smartphone).

Por último, el emprendimiento digital se identifica con nuevos productos y servicios basados en internet. Estos servicios se ejecutan solo en la nube, utilizando big data o inteligencia artificial. En este tipo de empresas, la tecnología es un factor de entrada, se persigue el alto crecimiento y despuntar sobre los competidores. Las fuentes de financiación son las mismas que en las empresas anteriores (ejemplo, Snapchat).

El presente estudio incluye las tres tipologías de empresas, con lo que tendremos en cuenta las particularidades de cada una de ellas, en lo referente a necesidades de financiación y requisitos tecnológicos. Se toma la perspectiva similar a la del informe extenso sobre el emprendimiento digital publicado por la Comisión Europea en 2015 (Bogdanowicz, 2015), en el que se hace énfasis sobre la innovación en tecnologías de la información de las empresas digitales, mientras se incluye un campo amplio de tecnologías. Este trabajo ofrece asimismo una clara afirmación sobre las diferencias entre emprendimientos tradicionales y digitales. En particular haremos referencia a los retos específicos a los que se enfrentan éstos últimos en la siguiente sección.

En términos generales, se puede resumir que las principales necesidades de estas empresas de base tecnológica son las relacionadas con la financiación y la comercialización de sus productos o servicios (Souto, 2012), aunque revisaremos las particularidades de empresas digitales en mayor detalle más adelante. Trataremos de analizar, por un lado, las barreras a las que habitualmente se enfrentan este tipo de empresas en su fase de creación y, posteriormente, las barreras más comunes a las que se enfrentan las mujeres emprendedoras.

El foco sobre el emprendimiento digital femenino encuentra su justificación por dos vertientes. En primer lugar, ya hemos podido ver que se trata de un sector con alto potencial de crecimiento y de impacto sobre la economía y la sociedad, tanto por su capacidad transformadora como su potencial de creación de empleo y orientación social. En segundo lugar, se trata de un sector tradicionalmente más dominado por hombres, por 
lo que conviene ahondar en la comprensión de las causas de esta disparidad para tener un impacto social mediante la mayor inclusión.

\section{Principales barreras al emprendimiento digital}

Como hemos podido establecer anteriormente, las empresas fundadas en el ámbito digital que se basan en tecnologías de la información, son diferentes de las startups que no hacen uso de estas tecnologías para su creación de valor. Resulta lógico pensar que los retos a los que se enfrentan unas y otras tampoco serán los mismos. Según el informe Emprendimiento Digital: Barreras y Drivers (Digital Entrepreneurship Barriers and Drivers por Bogdanowicz, 2015), los emprendimientos digitales sufren de la siguiente lista de barreras específicas:

- Marco regulador: al tratarse de empresas innovadoras, muchas de ellas ofrecen bienes y servicios que plantean retos novedosos al marco regulador, dificultando así su implementación. Un ejemplo popular de esto es la plataforma de viajes, Uber.

- Condiciones del mercado: muchas de las startups digitales operan en un mercado en el que se impone un estándar y las ventajas de ser primero pesan mucho. El riesgo de la absorción o de la imitación por un rival más potente suponen un problema para muchas empresas nacientes, además de ciertas limitaciones a la hora de acceder a mercados internacionales, debido a marcos legales diferentes (aun siendo su modelo de negocio de origen global - born globals).

- Acceso a la financiación: mientras que esta es una barrera comúnmente citada para empresas de reciente creación, en el caso de las digitales se potencia. El uso de tecnologías novedosas aumenta el riesgo percibido para los inversores y dificulta que éstos encuentren fondos. Cabe matizar esto por la buena fama de la que han disfrutado las empresas digitales últimamente y de los conocidos casos de éxito, como Alibaba, Uber o PayPal. Sin embargo, éstos son casos extraordinarios que no describen la mayoría.

- Capacidades emprendedoras: debido a la elevada importancia de las TIC, este tipo de emprendimientos requieren una combinación particular de capacidades, entre las cuales se deben hallar tanto un dominio tecnológico como capacidades relacionadas con la creación, la dirección de empresas y la sensibilidad social.

- Juventud del emprendedor: debido a que las tecnologías usadas son relativamente recientes, puede darse el caso de que la persona emprendedora sea joven y sufra discriminación debido a su edad a la hora de buscar apoyos o de captar clientes.

Como podemos ver, Bogdanowicz (2015) enumera con mucha claridad aquellas dificultades que están especialmente presentes entre empresas digitales, dejando claro que no son iguales que otro tipo de emprendimientos. Esta particularidad, además de su ya mencionado alto impacto en el desarrollo económico, justifican una atención particular sobre este tipo de startups. Seguidamente se analizará qué a qué clase de barreras específicas para mujeres emprendedoras hace referencia la literatura.

\section{Principales barreras de las emprendedoras}

Las barreras típicas para el emprendimiento identificadas por trabajos previos (GEM 2017 y 2018, Doing Business 2020, GEDI 2019) son el acceso a la financiación y la información, la complejidad del marco legal, el apoyo social a la figura del emprendedor o el acceso a canales de distribución y redes de contacto, entre otros (Jain \& Saiyed-Wajid, 2013). Éstas son las mismas para todo tipo de emprendimientos, pero afectan de forma diferente a diferentes tipos de empresas, dependiendo de las características de la persona emprendedora y de las propias startups. Estas dos características pueden agruparse en los factores internos que afectan al emprendimiento, a los que se suma el grupo de factores externos, que serán aquellos que apoyen o dificulten el emprendimiento dependiendo de la configuración del contexto socioeconómico en el que se crea la startup (Jain \& Saiyed-Wajid, 2013). Sin embargo, está claro que el contexto tiene gran influencia sobre la persona emprendedora, ya que puede formar a esta o apoyarla más implícitamente, mediante un alto reconocimiento social de la figura del emprendedor/a.

Se ha podido ver que, dependiendo del perfil de empresa que se analice, como podría ser una de mayor o menor uso tecnológico, más o menos dependiente de financiación o el grado de internacionalización de esta, ciertas dificultades tienen mayor peso de que otras. Por otro lado, el perfil de la persona que inicia el proyecto supone el otro condicionante para que ciertas dificultades sean más importantes que otras. Así, las emprendedoras sufren ciertas dificultades únicamente debido a su condición de mujer. 
Se revisan en lo que sigue las barreras externas e internas, donde las primeras hacen referencia a las condiciones del entorno en el que se emprende (el ecosistema de emprendimiento) y las segundas a las características específicas de las emprendedoras (rol social, capacidades y confianza en sí misma).

\section{a) Barreras externas}

Como se pudo ver, una de las principales barreras a la hora de establecer un negocio de base tecnológico suele ser el acceso a financiación ajena, muchas veces fruto de la novedad del producto ofrecido o de la falta de experiencia empresarial del emprendedor (Storey y Tether 1998). El hecho de que en este tipo de empresas predominen entre sus activos los elementos intangibles influye en esta mayor dificultad al acceso a la financiación. Las mujeres son las más afectadas por esta situación. En su estudio, The Boston Consulting Group, muestra como las mujeres creadoras de startups, pese a reportar un beneficio mayor en media que los hombres, suelen recibir menor financiación (Abouzahr et al., 2018). Se observa también cierta preferencia de los inversores hombres a financiar propuestas presentadas por hombres frente a los realizados por mujeres, aun cuando el contenido es el mismo (Brooks et al., 2014 para el caso de EE. UU.).

Cabe destacar que esto guarda una estrecha relación con la baja presencia de mujeres en firmas de capital riesgo (Smith, 2019) y con la posible preferencia de los inversores de conceder financiación a personas de su mismo género (Brooks et al. 2014). De acuerdo con la firma de medios e información Axios, solo el 9\% de los tomadores de decisiones en este tipo empresas financiadores en EE. UU. son mujeres, reduciendo así las posibilidades de acceso a financiación de las emprendedoras. Para España este porcentaje es aún menor, con solo un 6\% de mujeres entre los cargos de responsabilidad en las empresas de capital riesgo (Sekulits, 2020). A su vez, al no haber tantos antecedentes de éxito femeninos como masculinos (Observatorio Mujeres, Ciencia e Innovación, 2020), las empresas de capital riesgo y los inversores privados podrían mostrase más reticentes a la hora de apostar por mujeres emprendedoras (Fundación Innovación de la Economía Social, 2011).

\section{b) Barreras internas}

En segundo lugar, existen barreras a nivel personal, es decir, de la propia mujer emprendedora. Por un lado, se ha mostrado que el rol en la sociedad juega un papel importante, donde las mujeres tienden a dedicar más horas que los hombres a las actividades familiares, lo que conlleva una desigualdad de oportunidades profesionales (Straub, 2007). Al disponer de menos tiempo, pueden dedicar menos horas a la labor de emprender, que suele simultanearse con un trabajo por cuenta ajena. Asimismo, no desarrollan redes de contacto que resultan clave en el proceso de emprendimiento. Finalmente, el rol asignado en la familia crea una identidad propia que influye sobre la autoestima a la hora de emprender y sobre el apoyo de su entorno social (Global Entrepreneurship Research Association (GERA), 2017; Koellinger et al., 2013). El hecho de tener que jugar el rol de bread maker, ya sitúa a la mujer en una posición de desventaja antes de emprender, debido a sus menores ingresos. Esto supone un menor acceso a capital semilla (el necesario para comenzar un proyecto de emprendimiento desde cero) y menor capacidad de endeudamiento. Debido a estas causas, las mujeres se ven obligadas a emprender con menor financiación inicial y sufren la problemática de acceso a la financiación de forma más acentuada (García Villalobos et al, 2019). A esto hay que añadirle unas tasas de propiedad de inmuebles tradicionalmente más bajas que las de los hombres (Arekapudi \& AlmodóvarReteguis, 2020), empeorando aún más esta problemática.

Por otro lado, se muestra cómo las mujeres pueden llegar a tener una mayor autoexigencia, imponiéndose a sí mismas unos estándares más elevados, lo cual podría llevarlos, en ocasiones, a no emprender o tomar decisiones que impliquen un alto riesgo (Adams y Funk, 2012, Koellinger et al., 2013). De acuerdo con el GEM (2019) las mujeres puntúan más bajo en aquellas percepciones que facilitan el comportamiento emprendedor, como son la confianza en las habilidades para emprender y el conocimiento de otros emprendedores. Por otro lado, obtienen mayor puntuación en otro aspecto que inhibe la actitud emprendedora como el miedo al fracaso. Se ha podido ver que el mayor miedo al fracaso por parte de las mujeres ha llevado a una propensión de emprender menor que en el caso de los hombres (Koellinger et al., 2013).

\section{Objetivos y metodologías}

Los estudios publicados sobre el emprendimiento femenino hasta la fecha centran, en su gran mayoría, su atención en la problemática general, pero no se han centrado en el tipo de empresa digital, y siendo aún menos los trabajos que analizan el emprendimiento tecnológico femenino. El presente estudio describe el estado en el que se encuentra el emprendimiento digital en España, tratando de conocer las características 
sociodemográficas de las mujeres, sus motivaciones para el emprendimiento y las principales barreras y retos a los que se enfrentan. Mediante un estudio empírico se busca contrastar las barreras comúnmente citadas en la literatura para emprendimientos digitales con los emprendimientos digitales promovidos por mujeres. Así pues, se analizan los resultados de una encuesta enviada a 326 y contestada por 161 emprendedoras digitales ${ }^{7}$. Esto nos da una tasa de respuesta del 49,4\%.

La encuesta se realizó de forma telemática entre los meses de marzo-abril de 2020. Hay que mencionar que se trata de un periodo temporal particular, ya que coincidió con el comienzo de la crisis sanitaria del COVID-19 en España y el consiguiente periodo de cuarentena sanitaria. Esto se trató de atender mediante una pregunta específica en el cuestionario sobre el efecto esperado de la pandemia, y se considera que la mayoría de las preguntas no deberían verse afectadas por esta situación. Aun así, es posible que haya influido sobre el estado de ánimo de las encuestadas, introduciendo cierto sesgo.

El cuestionario estaba formado por 45 preguntas que se agrupaban en cuatro bloques: perfil de las empresas digitales fundadas por mujeres, principales características sociodemográficas de las emprendedoras, motivaciones para el emprendimiento y principales barreras, retos y necesidades. Este cuestionario fue testado y revisado antes de su publicación y los datos fueron procesados posteriormente usando IBM SPSS Statistics 24. En el siguiente punto se presentarán los resultados obtenidos tras un análisis de los estadísticos descriptivos.

\section{Resultados}

\subsection{Caracterización de las empresas}

El 39\% de las empresas encuestadas se encuentra en una fase en la que ya genera sus propios beneficios, mientras que un $42 \%$ todavía se encuentra en una fase muy temprana, gestionando el capital semilla. La facturación es, generalmente, reducida, no llegando al millón de Euros (92\%), de hecho, encontramos una parte importante por debajo de los $€ 100.000(72 \%)$. Si analizamos el tamaño de las empresas creadas por su inversión en activos, vemos el mismo comportamiento. Prácticamente todas $(96,2 \%)$ se sitúan por debajo del millón y la gran mayoría (81\%) no llega a los €100.000 de inversión.

Tabla. 1. Perfil de empresas

\begin{tabular}{|l|r|l|r|l|l|r|r|r|}
\hline \multicolumn{2}{|c|}{ Facturación último año } & \multicolumn{2}{l|}{ Inversión en Activos } & \multicolumn{2}{l|}{ Inversión en I+D } & \multicolumn{2}{l|}{ Financiación } \\
\hline Rango* & Distribución & Rango** & Distribución & Rango* & Distribución & Rango* & Distribución \\
\hline$<=100$ & $70 \%$ & $<1$ & $96 \%$ & $<=20$ & $50 \%$ & $<200$ & $73,9 \%$ \\
\hline $100-200$ & $9 \%$ & $1-2$ & $2 \%$ & $20-40$ & $13 \%$ & $200-400$ & $5,6 \%$ \\
\hline $200-300$ & $2 \%$ & $2-3$ & $1 \%$ & $40-60$ & $6 \%$ & $400-600$ & $5,0 \%$ \\
\hline $300-400$ & $3 \%$ & $>6$ & $1 \%$ & $60-80$ & $7 \%$ & $600-800$ & $3,1 \%$ \\
\hline $400-500$ & $2 \%$ & & & $80-100$ & $1 \%$ & $>800$ & $12,4 \%$ \\
\hline$>600$ & $14 \%$ & & & $100-120$ & $6 \%$ & & \\
\hline & & & & $>120$ & $18 \%$ & & \\
\hline
\end{tabular}

Fuente: Elaboración propia

Podemos observar que la gran mayoría declara un gasto en I+D (83\%), por reducido que sea. La mayor parte de este $83 \%$ (87\%) se concentra en la franja de hasta $€ 20.000$ de gasto, con un gasto medio de $€ 8.166$. Si consideramos que la facturación de la gran mayoría no llega a los $€ 100.000$, podemos observar una intensidad de I+D media (Inversión en I+D media/Facturación media) muy elevada del 28,5\%. Si comparamos estas cifras con las generales de las NEBTs, vemos que la Fundación MadrI+D describe en su último informe sobre estas empresas (Díaz \& Cid, 2019) la media de inversión en I+D de €76.000 y que un $21 \%$ excede los $€ 100.000$

7 El cuestionario fue enviado a mujeres emprendedoras en el ámbito digital a través de la asociación W Startup Community, cuya finalidad principal es el apoyo a mujeres al emprendimiento en el ámbito STEMB. Para ello se firmó un acuerdo de colaboración entre la Universidad CEU San Pablo, en la que trabajan los autores, y WStartupC. 
Gráfico. 1. Principales sectores de emprendimiento digital femenino

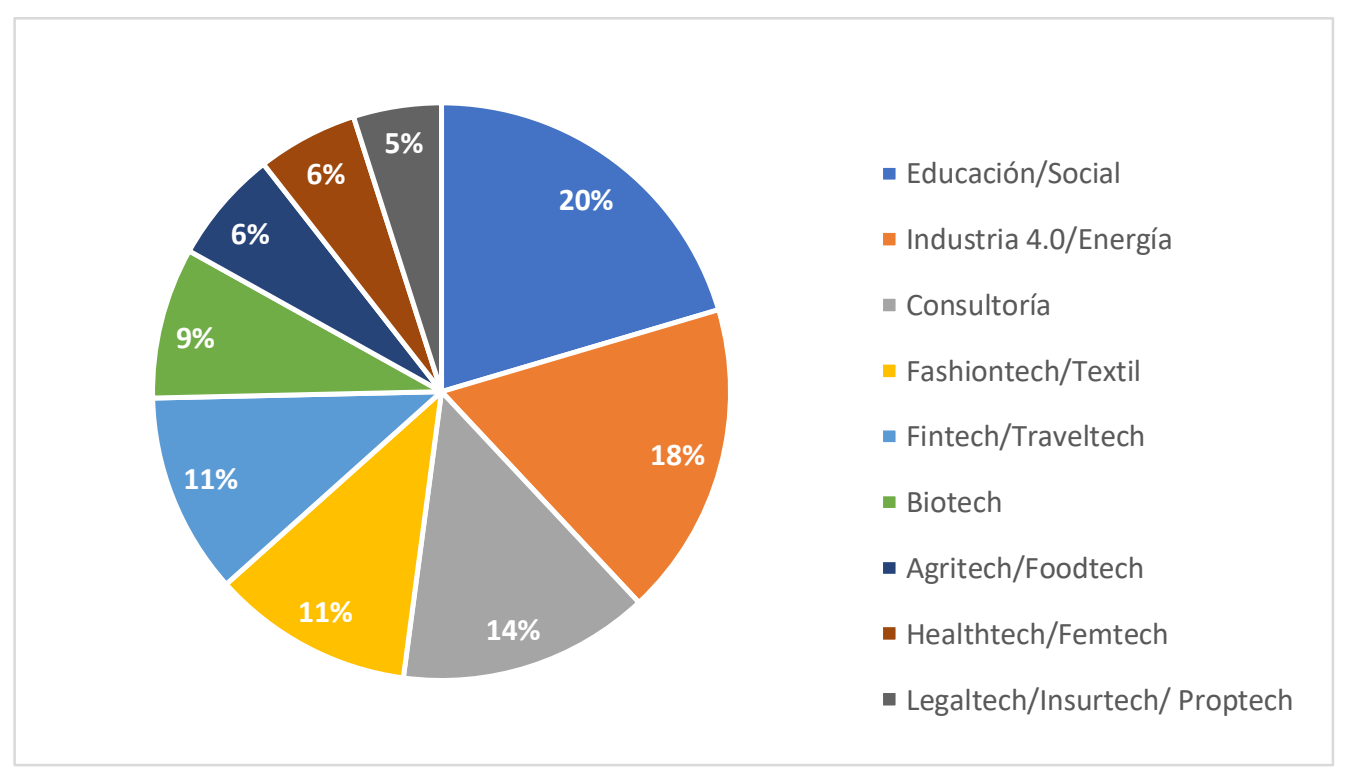

Fuente: Elaboración propia.

El ámbito de mayor preponderancia del emprendimiento digital femenino es el de la educación/social, según declaran las propias empresas analizadas. Por tanto, el impacto de estas empresas en actividades de componente social es significativo, siendo seguidas por la industria 4.0/energía, consultoría y, a partes iguales fashiontech y fintech. No obstante, surge la dificultad de encuadrar algunas de las primeras, dado que las mismas se integran también en alguno de los sectores identificados por razón de la actividad específica (textil, agrario...), además del grupo "Social" donde prefieren ser encuadradas por razón de su compromiso con el entorno en el que se ubican, de las personas que trabajan en ellas o de los públicos que atienden.

En lo referente a la financiación obtenida por las emprendedoras y sus orígenes, con independencia del sector de ocupación, se puede apreciar que fue generalmente baja, registrando un 73,9\% menos de $€ 200.000$ de inversión captada hasta el momento del estudio (edad media de las empresas 8,7 años). Si recordamos la baja cifra de inversión en activos y el reducido tamaño de las empresas, no resulta sorprendente que su inversión inicial tampoco fuera muy elevada.

Gráfico. 1. Principal fuente de financiación

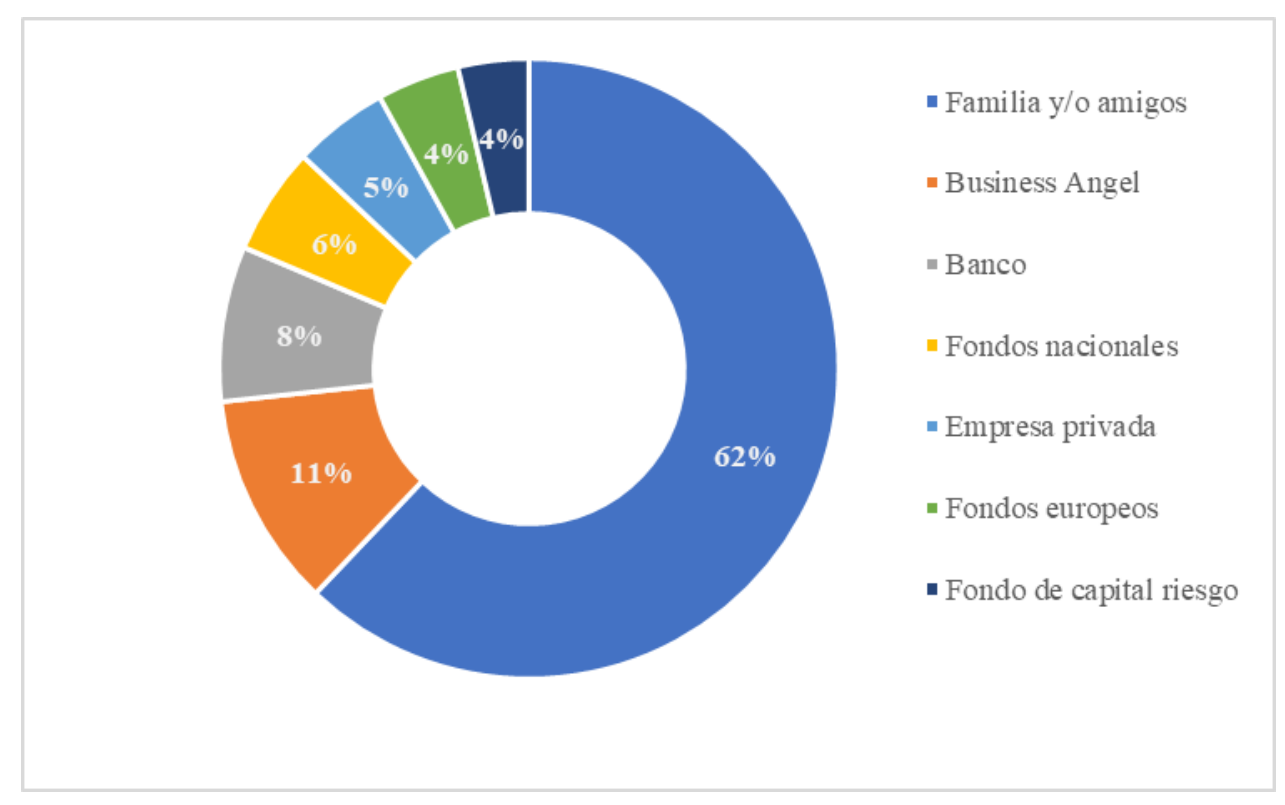

Fuente: Elaboración propia. 
La fuente principal fue, como en la mayoría de los emprendimientos, la familia y los amigos de la emprendedora (62,1\%). En solo un 11,2\% intervino un Business Angel y en tan solo un $8 \%$ un banco. Asimismo, en mucho menor medida se obtuvo financiación de otra empresa privada (5\%), de fondos públicos nacionales $(5,6 \%)$ o europeos $(4,3 \%)$. La participación de fondos de capital riesgo era la menor $(3,7 \%)$.

Para aquellas que obtuvieron fondos de un Business Angel, todos, excepto uno fueron hombres. Por un lado, podemos interpretar este resultado como positivo, ya que no observamos una problemática de género, que pudiera hacer pensar que será más probable que una mujer reciba financiación de otra mujer que de un hombre (Brooks et al., 2014), sin que se aprecie discriminación alguna por motivo del sector de actividad económica emprendida.

Gráfico. 2 Motivaciones para Emprender

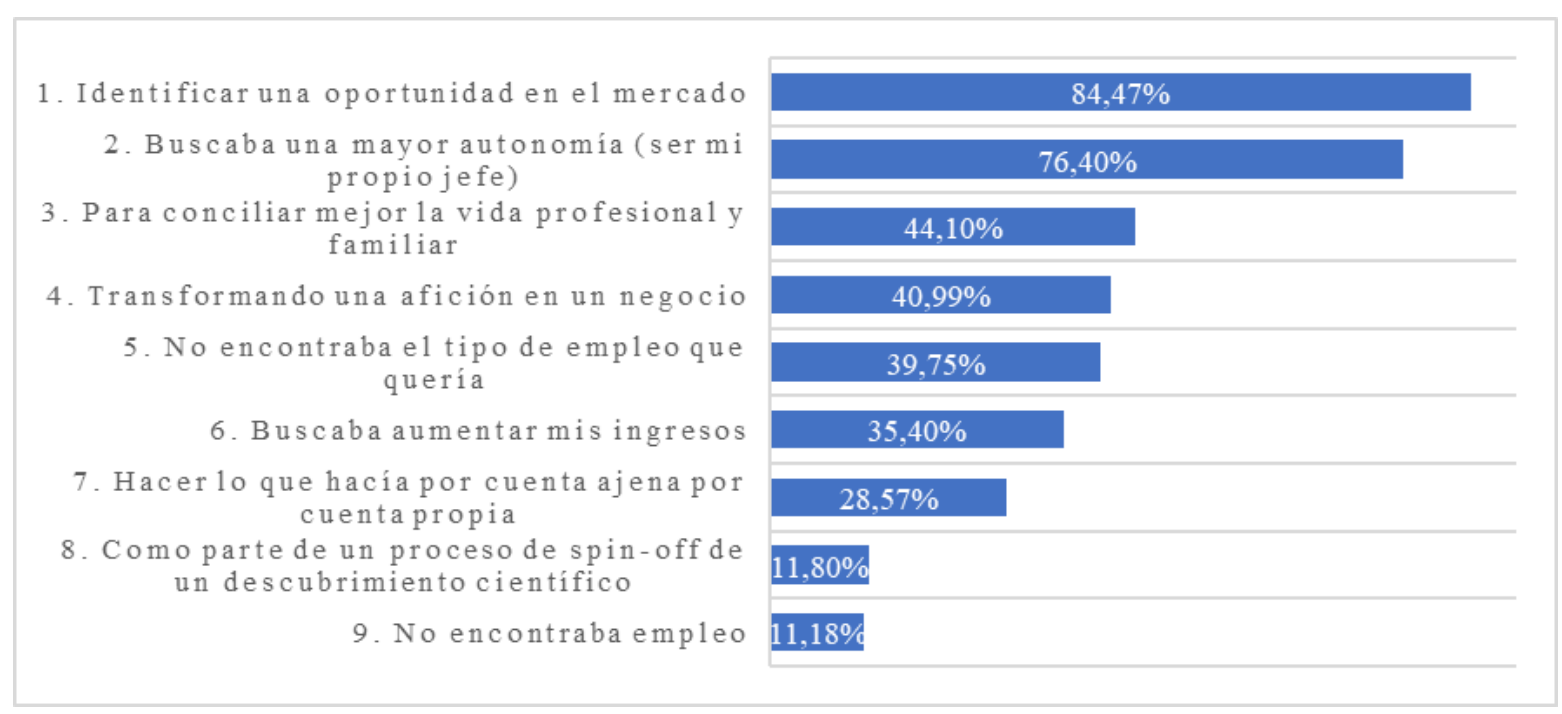

Fuente: Elaboración propia.

En términos globales podemos decir que los emprendimientos femeninos digitales en España estudiados fueron de oportunidad y no de necesidad. Es decir, las emprendedoras identificaron una oportunidad en su entorno y se encaminaron a crear una empresa para su aprovechamiento. No se trataba, por lo tanto, de una forma de buscar el sustento vital, ni por falta de oportunidades en el mercado laboral. Esto está en línea con el perfil de la economía española identificado por el Global Entrepreneurhsip Monitor - GEM (2017), y clasificado como impulsada por la innovación (innovation driven). Asimismo, responde al perfil general del tipo de emprendimiento que podemos encontrar en nuestro país según la última edición de este informe, que se describe como aquel que busca mejorar la situación vital actual, aumentando la autonomía y/o los ingresos (Improvement-driven opportunity) (GEM 2019). Este tipo de emprendimiento casi duplica aquellos emprendimientos que son motivados por necesidad en España. Finalmente, los emprendimientos digitales, debido a sus características particulares de uso de la tecnología y el perfil de sus fundadores, suelen ser por oportunidad, si bien en el caso de los sociales, sean o no digitales, se han revelado con cierta frecuencia una alternativa necesaria para los colectivos que los fundan o sus trabajadores que por sus características (discapacidad, minusvalía; etc.) pueden tener más dificultades en el acceso al empleo.

En lo personal, aproximadamente un tercio (28\%) declaró que no se inspiró en ningún referente a la hora de emprender, aunque un $26,1 \%$ cita a alguno de sus padres u otro familiar (ver gráfico más abajo). Asimismo, otras emprendedoras sirvieron de referente a un $23 \%$, dato que se solapa con la figura de un/a empresario/a nacional (18\%) o internacional $(17,4 \%)$. Por el contrario, una directora o profesor/a inspiró a la menor parte de las emprendedoras. 
Gráfico. 4. Influencia para emprender

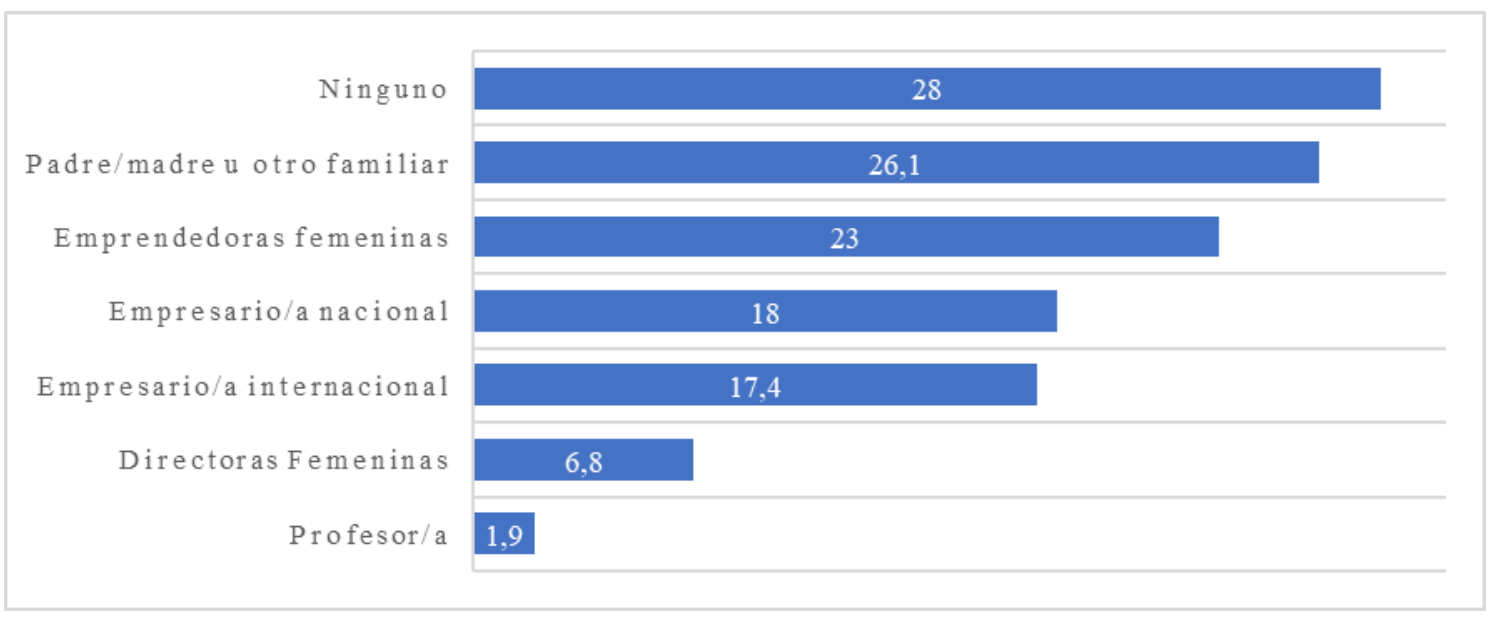

Fuente: Elaboración propia.

Estos resultados están en línea con estudios previos que muestran como los descendientes de emprendedores muestran una mayor probabilidad de crear su propio negocio (Fairlie y Bobb, 2007). Asimismo, se ha mostrado que tener modelos de referencia puede motivar a las personas a emprender, ya sea dándoles apoyo moral o consejos que puedan necesitar (Bosma \& Kelly, 2019). De acuerdo con el Global Entrepreneurship Monitor (2019) el porcentaje de la población emprendedora con un referente emprendedor es del $52,4 \%$, frente al $33,4 \%$ de la población no emprendedora.

\subsection{Principales barreras al emprendimiento digital femenino}

Para el caso de las emprendedoras digitales en España se pidió que se eligiese el Top 3 de los mayores retos a los que se han tenido que enfrentar a la hora de emprender. Podemos observar que el mayor reto a la hora de emprender fue el Acceso a la Financiación Temprana, con la Falta Inicial de Ingresos/Ahorros en segundo lugar y citándose al Marco Legal con más frecuencia en tercero (ver tabla más abajo). Resultaría lógico pensar que los primeros dos conceptos están íntimamente ligados, ya que la financiación temprana va, en gran medida, dedicada al pago de salarios, entre los que se cuenta el de la emprendedora.

Tabla. 2. Mayores retos para emprender

\begin{tabular}{|l|l|l|l|}
\hline & 1 & 2 & 3 \\
\hline Acceso a financiación temprana & $22 \%$ & $10 \%$ & $11 \%$ \\
\hline La falta inicial de ingresos / ahorros & $15 \%$ & $18 \%$ & $13 \%$ \\
\hline El marco legal para iniciar el negocio & $12 \%$ & $15 \%$ & $14 \%$ \\
\hline Conocimiento sobre el proceso de creación y gestión empresarial & $17 \%$ & $12 \%$ & $8 \%$ \\
\hline Encontrar socios & $8 \%$ & $8 \%$ & $12 \%$ \\
\hline Conocer los gustos de mis clientes & $8 \%$ & $9 \%$ & $8 \%$ \\
\hline Conocimiento técnico sobre el producto / servicio a ofrecer & $6 \%$ & $8 \%$ & $10 \%$ \\
\hline Encontrar proveedores & $5 \%$ & $6 \%$ & $8 \%$ \\
\hline Conciliación con la vida familiar & $4 \%$ & $1 \%$ & $2 \%$ \\
\hline Acceso a canales de distribución & $3 \%$ & $8 \%$ & $9 \%$ \\
\hline Acceso a servicios complementarios & $1 \%$ & $5 \%$ & $5 \%$ \\
\hline
\end{tabular}

Fuente: Elaboración propia.

La búsqueda de socios y conocer los gustos de los clientes se sitúan a mitad de la tabla, seguidos por el conocimiento técnico. Considerando esto, junto con el conocimiento sobre la creación de empresa como reto mayor, nos hace pensar que la mayoría de las emprendedoras son especialistas en su ámbito, pero que la creación de la empresa en sí supuso un reto. Cabe destacar que la conciliación con la vida familiar aparece muy abajo en la tabla, no dándose aquí una asunción común en el emprendimiento femenino, que explica la menor tasa de emprendimiento entre mujeres por su rol en la familia, como se comentó anteriormente. Esto 
se evidencia más explícitamente cuando se les pregunta directamente por esta cuestión y casi un 70\% afirma que su rol en la familia no les ha supuesto un problema a la hora de emprender (ver gráfico más abajo). Aquí hay que considerar que la mayoría de las emprendedoras no tienen hijos y el gran apoyo familiar que afirman haber recibido. Finalmente, el acceso a canales de distribución y a servicios complementarios no fueron problemas a la hora de emprender.

Gráfico. 5. Rol en la familia e impacto en el emprendimiento

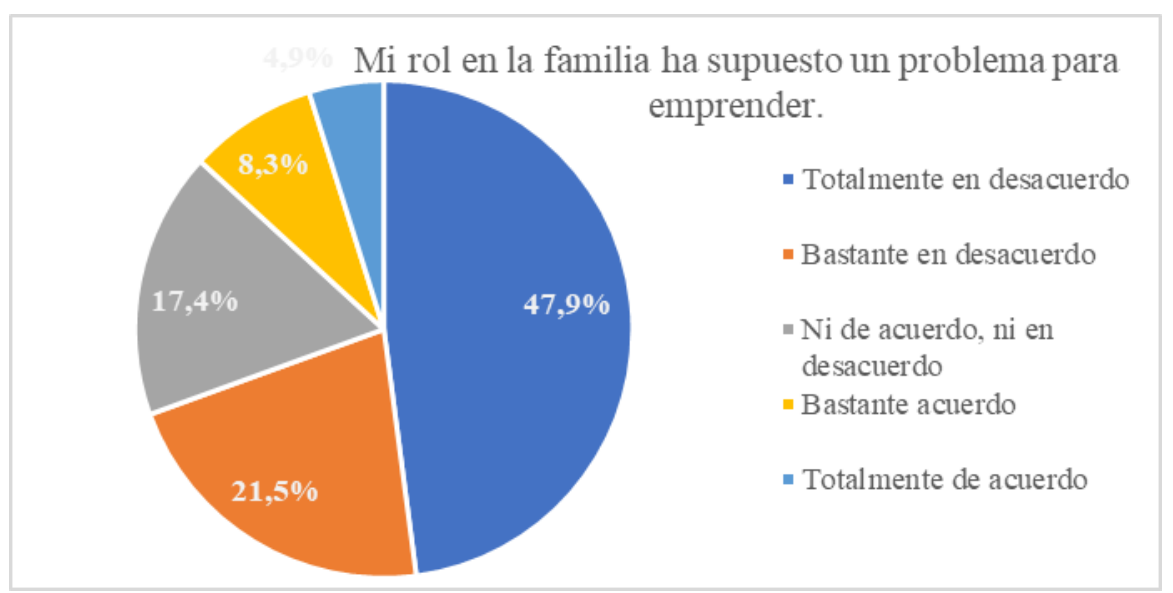

Fuente: Elaboración propia.

El acceso a redes de contacto, que frecuentemente es citado como una barrera más elevada para mujeres que para hombres (Hanson \& Blake, 2009; Sappleton, 2009), tampoco parece haber sido un impedimento para casi la mitad de ellas $(48,5 \%)$.

En principio, podemos resumir que las mayores barreras para las emprendedoras digitales en sus negocios de base o no social son la falta de fondos al inicio de su proceso de emprendimiento, un marco legal complicado y unos conocimientos incompletos sobre la creación y gestión de empresas.

Si buscamos el detalle de la barrera de la financiación, vemos que para la mayor parte de las emprendedoras (60\%) no ha sido fácil acceder a fuentes de financiación temprana (capital semilla). Mientras que el acceso a financiación temprana fue complicado, la financiación para el crecimiento del negocio fue más fácil. Un $45 \%$ afirma que ha sido relativamente fácil, mientras que un $27 \%$ denota complicaciones en este punto. Esta situación se ha visto agravada por la crisis del COVID-19, valorándose el impacto de ésta sobre la facilidad de encontrar financiación con un 4,3, siendo 5 la mayor dificultad.

Desde un punto de vista interno, cabe destacar que la gran mayoría de las emprendedoras $(82,6 \%)$ sentían que tenían las capacidades suficientes para emprender, lo que contrasta con estudios previos en los que las mujeres mostraban niveles menores de confianza en sus capacidades para el emprendimiento (Global Entrepreneurship Research Association (GERA), 2017; Koellinger at al., 2013) 
Gráfico. 6. Género e impacto en el emprendimiento

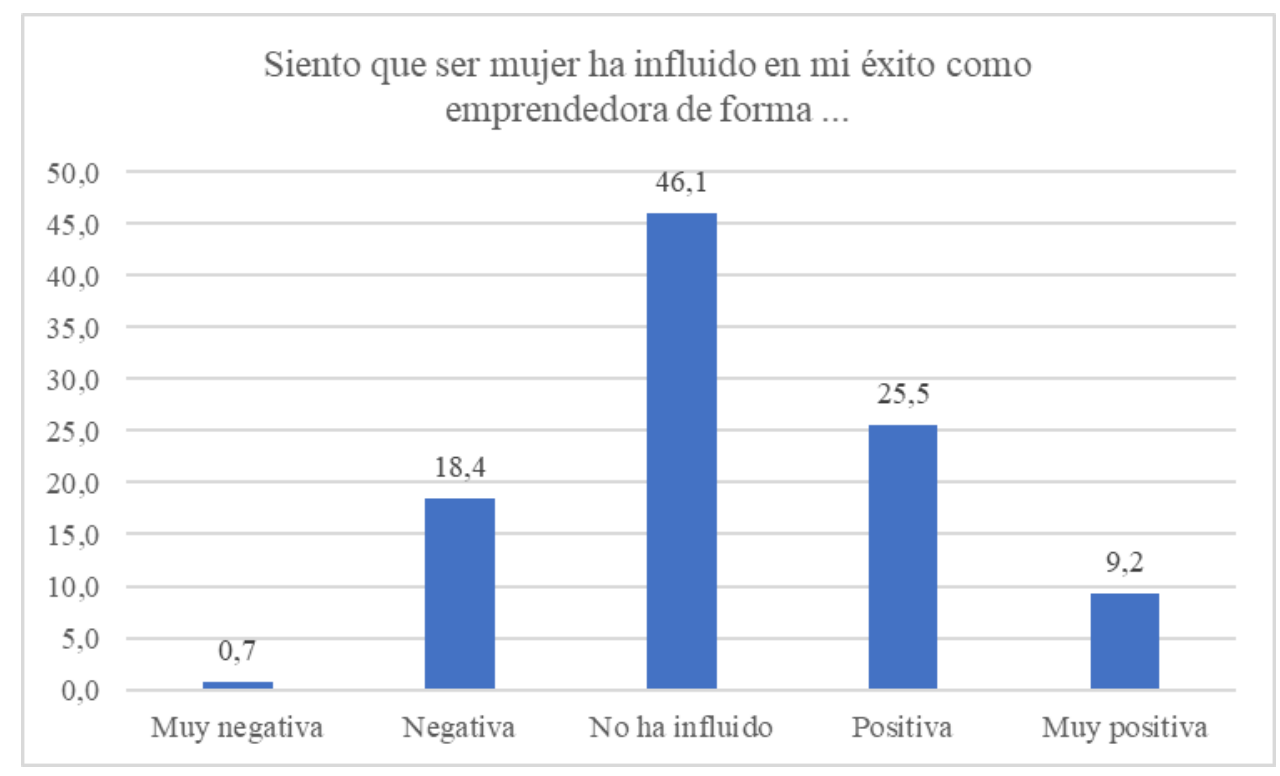

Fuente: Elaboración propia.

En general, el hecho de ser mujer no ha influido en ningún sentido en el emprendimiento para el 46,1\%, influyendo en sentido positivo o muy positivo para un $25,5 \%$ y $9,2 \%$, respectivamente. Por el contrario, un $18,4 \%$ piensa que influyó negativamente.

Si nos centramos en el ámbito digital, hay un $23 \%$ que afirma que su sector es más exigente para una mujer que para un hombre y un 19\% que está bastante de acuerdo con esta afirmación. Por el contrario, la mayoría no parece considerar el género como dificultad, aunque tampoco se oponen a la aserción.

\section{Conclusiones}

Podemos confirmar para el caso español que las barreras específicas de empresas digitales fundadas por mujeres están presentes. La orientación social de la empresa no varía a la hora de establecer estás limitaciones existentes, bien sea por la falta de financiación y de ingresos iniciales o de un marco legal claro en el proceso de puesta en marcha.

Sin embargo, las particulares dificultades de las mujeres emprendedoras, como su rol en la familia y la sociedad o la discriminación por género, no se pudieron confirmar. El rol en la familia no supuso un problema para la mayoría de las emprendedoras encuestadas. El hecho de ser mujer fue una complicación añadida para una parte relativamente reducida de la muestra, aunque sí parece percibirse el sector como más exigente para las mujeres que para los hombres. Esto confirma las conclusiones del estudio llevado a cabo por Sappleton (2009), en el que concluye que es más difícil desarrollar relaciones en sectores dominados por hombres, aunque el género de la persona emprendedora no resultó determinante.

No se pudo encontrar evidencia de discriminación por género a la hora de recibir financiación, ni tampoco porque el negocio tuviese más o menos vocación social, aunque aquí el estudio debe reconocer su limitación por su sesgo del superviviente (no se consultó por qué no obtuvieron financiación). Las emprendedoras que obtuvieron financiación no la obtuvieron mayoritariamente por mujeres, sino más bien al contrario, por hombres.

Aunque las dimensiones de falta de financiación (bajo acceso a financiación y falta de ingresos) y el marco legal suponen los dos factores más citados como barreras, la falta de conocimientos específicos se cita en tercer lugar. Esto confirma la barrera específica para empresas digitales citada en la literatura (Bogdanowicz, 2015), donde el dominio tanto de conocimientos técnicos avanzados, junto con los específicos de creación y gestión empresarial no siempre se encuentran en la misma persona.

A la hora de referirnos a políticas de promoción del emprendimiento femenino conviene fijarse, además de los condicionantes facilitadores del entorno, en los factores que motivaron el emprendimiento a nivel personal. Como hemos podido observar en el estudio, los familiares y emprendedoras nacionales e internacionales fueron importantes fuentes de inspiración, pero también el interés por el desarrollo rural, el empleo de calidad y la sostenibilidad. 


\section{Limitaciones y estudios futuros}

El presente estudio sufre la limitación de sesgo del superviviente, debido a que sólo se encuestaron a empresas fundadas activas y no aquellas que no lograron sobrevivir. Eso también se refiere a ciertas cuestiones específicas del estudio, como son las empresas que han recibido financiación, ya que no se incluyó una pregunta sobre por qué no recibieron financiación. Debido a que las razones subyacentes a esta decisión no suelen ser conocidas por las emprendedoras, no parecía lógica incluir una pregunta así en el cuestionario.

En segundo lugar, el estudio puede sufrir sesgos cognitivos de las personas que lo responden, debido a que muchas cuestiones solicitan la evaluación subjetiva de la persona encuestada. Exponiendo la situación extraordinaria de la pandemia de COVID-19 en la descripción metodológica, se trató de alertar sobre la posible influencia de un evento tan grave sobre las respuestas. Aun así, se considera que esta dificultad es asumida por muchos estudios que siguen la misma técnica, tal como el Global Entrepreneurship Monitor.

Finalmente, el estudio carece de una muestra de empresas digitales creadas por hombres, que habría permitido comparar las dificultades de las mujeres emprendedoras en el ámbito digital con las de los hombres.

Como estudios futuros se recomendaría profundizar en mayor medida en las barreras identificadas para poder encontrar sus causantes originales y poder ofrecer recomendaciones para paliarlas. Específicamente, las dificultades de financiación deberían estudiarse más detenidamente para comprender si se deben a la tecnología utilizada, características del proyecto emprendedor (orientación marcadamente social o no) o debilidades del ecosistema de emprendimiento. Asimismo, sería interesante conocer qué partes del marco legal son las que dificultan más la creación de las empresas digitales fundadas por mujeres. Por último, se propone realizar un estudio comparativo con una muestra de empresas fundadas por hombres para poder identificar aún más las particularidades de las empresas digitales creadas por mujeres y si se confirma que son más proclives a la búsqueda del valor social en sus iniciativas emprendedoras.

\section{Referencias bibliográficas}

Acs, Z. J., Audretsch, D. B., Braunerhjelm, P., \& Carlsson, B. (2012) Growth and Entrepreneurship. Small Business Economics, 39(2), pp. 289-300. DOI:10.1007/s11187-010-9307-2.

Adams, R. B. \& Funk, P. (2012) Beyond the glass ceiling: Does gender matter? Management Science, № 58, pp. 219235.

Alguacil Marí, P., \& Román Cervantes, C. (2020) Presentación monográfico: La economía social ante sus retos: emprendimiento, transformación digital, género y desarrollo sostenible. REVESCO. Revista de Estudios Cooperativos, No 133, e69000, https://doi.org/10.5209/reve.69000.

Arekapudi, N., \& Almodóvar-Reteguis, N. L. (24 de Febrero de 2020) Let's Talk Development. Obtenido de World Bank: https://blogs.worldbank.org/developmenttalk/womens-property-rights-are-key-economic-development.

Asociación RED GEM España. (2020) Informe GEM España 2019-2020. Santander: Editorial de la Universidad de Cantabria.

Almus, M. \& Nerlinger, E. A. (1999) Growth of New Technology-Based Firms: Which Factors Matter?. Small Business Economics, $\mathrm{N}^{\circ} 13$, pp. 141-154.

Bogdanowicz, M. (2015) Digital Entrepreneurship Barriers and Drivers. The need for a specific measurement framework. Institute for Prospective Technological Studies. DOI:10.2791/3112.

Bosma, N., \& Kelly, D. (2019) Global Entrepreneurship Monitor: 2018/2019 Global Report. Santiago de Chile: Gráfica Andes.

Brooks, A. W., Huang, L., Kearney, S. W., \& Murray, F. E. (2014) Investors prefer entrepreneurial ventures pitched by attractive men. Proceedings of the National Academy of Sciences, $\mathrm{N}^{\mathrm{o}} 111$, pp. 4427-4431. online. Obtenido de http://doi.org/10.1073/pnas.1321202111.

Díaz, E., \& Cid, I. (2019) Caracterización y necesidades de las Nuevas Empresas de Base Tecnológica (NEBTs) en el periodo 2004-2018. Madrid: Fundación para el Conocimiento madri+d.

European Union Startups (2020, 1 de Abril 2020) https://www.eu-startups.com/.

Fernandez Guadaño, J., López Millán, M., Sarria Pedroza, J. (2020) Cooperative Entrepreneurship Model for Sustainable Development. Sustainability, No 12, 5462.

Fundación Innovación de la Economía Social (2011) Barreras sociales de las mujeres para emprender. Málaga: Junta de Andalucía.

Galindo, M.-Á., \& Méndez-Picazo, M.-T. (2013) Innovation, entrepreneurship and economic growth. Management Decision, 51(3), pp. 501-514. DOI:10.1108/00251741311309625.

García Villalobos, J.C., Villaseca Morales, D., González Pérez, S. (2019) Emprendimiento femenino y financiación social: un estudio comparado. REVESCO, Revista de Estudios Cooperativos, Tercer Cuatrimestre, № 132 , pp. $97-$ 121. http://dx.doi.org/10.5209/REVE.65974. 
Giones, F., \& Brem, A. (2017) Digital technology entrepreneurship: A definition and research agenda. Technology Innovation Management Review, 7(5).

Global Entrepreneurship Research Association (GERA) (2017) Global Entrepreneurship Monitor 2016/2017. Chicago: Global Entrepreneurship Monitor

González Pernía, J. L., Martiarena, A. A., \& Peña Legazcue, I. (2012) Género y actividad exportadora de los emprendedores en España. Economía Industrial, No 383, pp. 91-110.

Hanson, S., \& Blake, M. (2009) Gender and Entrepreneurial Networks. Regional Studies, 43(1), pp. $135-149$. DOI:10.1080/00343400802251452.

Hernández Ortiz, M. J., García Martí, E., Martínez Jiménez, R., Pedrosa Ortega, C., \& Ruiz Jiménez, C. (2020) El efecto de la diversidad de género sobre el rendimiento de las sociedades cooperativas agroalimentarias españolas. REVESCO. Revista de Estudios Cooperativos, № 133, e67337. https://doi.org/10.5209/reve.67337.

Hernández Ortiz, M. J., Ruiz Jiménez, C., García Martí, E., \& Pedrosa Ortega, C. (2018) Situación actual de la igualdad de género en los órganos de gobierno de las sociedades cooperativas agroalimentarias. REVESCO. Revista de Estudios Cooperativos, No 129, pp. 66-83. https://doi.org/10.5209/REVE.61933.

Justo, R. (2017) Emprendimiento sostenible en clave femenina. https://www.ie.edu/insights/es/articulos/emprendimiento-sostenible-clave-femenina.

Koellinger, P., Minniti, M., \& Schade, C. (2013) Gender Differences in Entrepreneurial Propensity. Oxford Bulletin of Economics and Statistics, 75(2), 0305-9049. DOI:10.1111/j.1468-0084.2011.00689.x.

Meyer, N., \& de-Jongh, J. (2018) The Importance of Entrepreneurship as a Contributing Factor to Economic Growth and Development: The Case of Selected European Countries. Journal of Economics and Behavioral Studies, 10(4), pp. 287-299. DOI:10.22610/jebs.v10i4.2428.

Minniti, M. (2010) Female Entrepreneurship and Economic Activity. The European Journal of Development Research, 22(3), pp. 294-312. DOI:10.1057/ejdr.2010.18.

Minniti, M. (2012) El emprendimiento y el crecimiento económico de las naciones. Economía industrial, 1(383), pp. 23-30.

Montero González, B. y Camacho Ballesta, J.A. (2018) Caracterización del emprendimiento femenino en España: una visión de conjunto. REVESCO. Revista de Estudios Cooperativos, Tercer Cuatrimestre, $N^{o}$ 129, pp. 39-65. DOI: 10.5209/REVE.61936.

Motohashi, K. (2005) University-industry collaborations in Japan: The role of new technology-based firms in transforming the National Innovation System. Research Policy, N³4, pp. 583-594.

Naldi, F., Luzi, D., Valente, A. and Parenti, I.V. (2005) Scientific and technological performance by gender, in Moed, H.F., Glänzel, W. and Schmoch, U. (Eds.): Handbook of Quantitative Science and Technology Research, pp.299314, Springer, Netherlands.

Observatorio Mujeres, Ciencia e Innovación (2020) Mujeres e innovación 2020. Madrid: Ministerio de Ciencia e Innovación.

Sánchez Espada, J.; Martín López, S.; Bel Durán, P. y Lejarriaga Pérez de las Vacas, G. (2018) Educación y formación en emprendimiento social: características y creación de valor social sostenible en proyectos de emprendimiento social. REVESCO. Revista de Estudios Cooperativos, Tercer Cuatrimestre, N ${ }^{\circ} \quad 129, \quad$ pp. 16-38. http://dx.doi.org/10.5209/REVE.62492.

Sappleton, N. (2009) Women non-traditional entrepreneurs and social capital. International Journal of Gender and Entrepreneurship, 1(3), pp. 192-218. DOI:10.1108/17566260910990892.

Sekulits, C. (2 de Febrero de 2020) Por qué hay tan pocas mujeres inversoras en 'start up'. Espansión. Obtenido de https://www.expansion.com/expansion-empleo/2020/02/10/5e3d3b74468aeb20288b45fa.html.

Souto, J. E. (2012) Innovación, emprendimiento y empresas de base tecnológica en España: factores críticos e impacto sobre la competitividad de la economía. Colección madri+d. A Coruña: Netbiblo.

Spain Startup-South Summit Informe. (2009) Mapa del emprendimiento 2019. Madrid.

Storey, D. J. y Tether, B. S. (1998) New technology-based firms in the European Union: an introduction. Research Policy (26) 9, pp. 933-946.

Straub, C. (2007) A comparative analysis of the use of work-life balance practices in Europe. Gender in Management, 22(4), 289.

Wong, P. K., Ho, Y. P., \& Autio, E. (2005). Entrepreneurship, Innovation and Economic Growth: Evidence from GEM data. Small Business Economics (24), pp. 335-350. 